connecting the fixed points of $T$. (4) If $O_{1}$ and $O_{2}$ are disjoint simply connected domains invariant under a loxodromic $T$, the corresponding arcs, as in (3), divide $S$ into two Jordan regions, one or the other of which must contain any domain disjoint from $O_{1}$ and $O_{2}$. (5) If $O$ is a simply connected domain invariant under an elliptic $T$, then $O$ must contain a fixed point of $T$.

The examples are elaborations of the ideas in L. R. Ford, Automorphic functions, 2nd ed., Chelsea, 1951, pp. 55-59.

Brown UNIVERSITY

\title{
DIFFERENTIABLE NORMS IN BANACH SPACES ${ }^{1}$
}

BY GUILLERMO RESTREPO

Communicated by W. Rudin, January 20, 1964

1. Introduction. In $[4$, p. 28] S. Lang has asked whether or not a separable Banach space has an admissible norm of class $C^{1}$. In this note we indicate a proof of the following theorem, which characterizes those Banach spaces for which such a norm exists.

Theorem 1. A separable Banach space has an admissible norm of class $C^{1}$ if and only if its dual is separable.

It follows from this theorem that not even $C(I)$ possesses an admissible differentiable norm.

2. Preliminaries. Let $X$ be a Banach space with norm $\alpha$; we write $S_{\alpha}=\{x \mid \alpha(x)=1\}$ and $B_{\alpha}=\{x \mid \alpha(x) \leqq 1\}$. A norm in $X$ is admissible if it induces the same topology as does $\alpha$. The dual space is written $X^{*}$ and the norm dual to $\alpha$ is denoted by $\alpha^{*}$. An $f \in X^{*}$ is called a support functional to $B_{\alpha}$ at $x \in S_{\alpha}$ if $\alpha^{*}(f)=f \cdot x$; if $f$ has norm 1 , it is called a normalized support functional and is written $\nu_{x}$. A norm is smooth if there is a unique normalized support functional to $B_{\alpha}$ at each $x \in S_{\alpha}$. The norm $\alpha$ is differentiable at $x \neq 0$ if there is an $\alpha^{\prime}(x)$ $\in X^{*}$ such that

$$
\lim _{y \rightarrow x ; y \neq x} \frac{\left|\alpha(y)-\alpha(x)-\alpha^{\prime}(x) \cdot(y-x)\right|}{\alpha(y-x)}=0
$$

\footnotetext{
${ }^{1}$ Research partially supported by NSF Grant G-24471.
} 
and a norm differentiable at each $x \in X-\{0\}$ is of class $C^{1}$ if the map $\alpha^{\prime}: X-\{0\} \rightarrow X^{*}$, defined by $x \rightarrow \alpha^{\prime}(x)$, is continuous. The following two results are well known:

1. Klee [3]. Let $X$ and $X^{*}$ be separable. Then there exists an admissible norm $\alpha$ in $X$ such that $\alpha^{*}$ is strictly convex, and such that whenever a sequence $\left\{f_{n}\right\}$ in $X^{*}$ converges to $f \in X^{*}$ in the $w^{*}$ topology, then $\alpha^{*}\left(f_{n}\right) \rightarrow \alpha^{*}(f)$ implies $\alpha^{*}\left(f-f_{n}\right) \rightarrow 0$.

2. Bishop-Phelps [1]. In any Banach space $X$, the set of all the support functionals to $B_{\alpha}$ is dense in $X^{*}$.

3. Proof of Theorem 1. It is not difficult to see that if the norm $\alpha$ is differentiable at $x \in S_{\alpha}$, then $\alpha^{\prime}(x)=\nu_{x}$ is a normalized support functional to $B_{\alpha}$ at $x$, and is unique. The map $x \rightarrow \nu_{x}$ of $S_{\alpha}$ into $S_{\alpha^{*}}$ is denoted by $\mu$. We first establish the following general theorem:

Theorem 2. (a) If $\alpha$ is a smooth norm in $X$, then the map $\mu$ is continuous when the norm topology is used in $X$ and the w*-topology is used in $X^{*}$.

(b) The norm $\alpha$ is of class $C^{1}$ if and only if the map $\mu$ is continuous in the norm topologies.

(c) A norm is of class $C^{1}$ if and only if it is differentiable at every point of $S_{\alpha}$.

Complete details will be published elsewhere; using this result, we prove Theorem 1 as follows:

Assume $X^{*}$ is separable, and let $\alpha$ be the norm of Klee's theorem. By a well-known duality, $\alpha$ is smooth. Theorem 2(a) assures $\mu$ is continuous with the $w^{*}$-topology in $X^{*}$, and then Klee's theorem shows $\mu$ is continuous in the norm topology. By Theorem 2(b), $\alpha$ is therefore of class $C^{1}$.

Assume now that $\alpha$ is of class $C^{1}$. Extend the continuous map $\mu$ to a continuous $\hat{\mu}: X-\{0\} \rightarrow X^{*}$ by setting $\hat{\mu}(x)=\alpha(x) \cdot \mu(x / \alpha(x))$. The image of $\hat{\mu}$ evidently contains the set of all the support functionals to $B_{\alpha}$, and an application of the Bishop-Phelps theorem shows at once that $X^{*}$ is separable whenever $X$ is separable.

\section{BiBLIOGRAPHY}

1. E. Bishop and R. R. Phelps, The support functionals of a convex set, Proc. Sympos. Pure Math., Vol. 7, pp. 27-35, Amer. Math. Soc., Providence, R. I., 1963. 1960.

2. J. Dieudonne, Foundations of modern analysis, Academic Press, New York,

3. V. Klee, Mappings into normed linear spaces, Fund. Math. 49 (1960), 25-63.

4. S. Lang, Introduction to differentiable manifolds, Interscience, New York, 1962.

University of SoUthern CaLIFornia 